\title{
CONGRUENCES MODULO POWERS OF 2 FOR FU'S 5 DOTS BRACELET PARTITIONS
}

\author{
ERNEST X. W. XIA and OLIVIA X. M. YAO ${ }^{\bowtie}$
}

(Received 20 April 2013; accepted 29 June 2013; first published online 22 August 2013)

\begin{abstract}
In 2007, Andrews and Paule introduced a new class of combinatorial objects called broken $k$-diamond partitions. Recently, Shishuo Fu generalised the notion of broken $k$-diamond partitions to combinatorial objects which he termed $k$ dots bracelet partitions. Fu denoted the number of $k$ dots bracelet partitions of $n$ by $\mathfrak{B}_{k}(n)$ and proved several congruences modulo primes and modulo powers of 2 . More recently, Radu and Sellers extended the set of congruences proven by Fu by proving three congruences modulo squares of primes for $\mathfrak{B}_{5}(n), \mathfrak{B}_{7}(n)$ and $\mathfrak{B}_{11}(n)$. In this note, we prove some congruences modulo powers of 2 for $\mathfrak{B}_{5}(n)$. For example, we find that for all integers $n \geq 0, \mathfrak{B}_{5}(16 n+7) \equiv 0\left(\bmod 2^{5}\right)$.
\end{abstract}

2010 Mathematics subject classification: primary 11P83; secondary 05A17.

Keywords and phrases: congruences, 2-dissections, partitions, $k$ dots bracelet partitions.

\section{Introduction}

This paper is concerned with congruences modulo powers of 2 for the number of 5 dots bracelet partitions. We find several arithmetic progressions $A n+B$ such that for any integer $n \geq 0$,

$$
\mathfrak{B}_{5}(A n+B) \equiv 0\left(\bmod 2^{k}\right)
$$

where $\mathfrak{B}_{5}(n)$ is the number of 5 dots bracelet partitions of $n$ and $k$ is a positive integer.

A combinatorial study guided by MacMahon's partition analysis led Andrews and Paule [1] to the construction of a new class of directed graphs called broken $k$ diamond partitions. A broken $k$-diamond partition $\pi=\left(a_{1}, a_{2}, \ldots ; b_{2}, b_{3}, b_{4}, \ldots\right)$ is a plane partition satisfying the relations illustrated in Figure 1, where here and in the rest of the figures, $a_{i}, b_{i}$ are nonnegative integers and $a_{i} \rightarrow a_{j}$ is interpreted as $a_{i} \geq a_{j}$. More precisely, each building block in Figure 1, except for the broken block $\left(b_{2}, b_{3}, \ldots, b_{2 k+2}\right)$, has the same order structure as shown in Figure 2. Each block is called a $k$-elongated partition diamond of length 1 , or a $k$-elongated diamond, for short.

This work was supported by the National Natural Science Foundation of China (11201188 and 11226300).

(c) 2013 Australian Mathematical Publishing Association Inc. 0004-9727/2013 \$16.00 

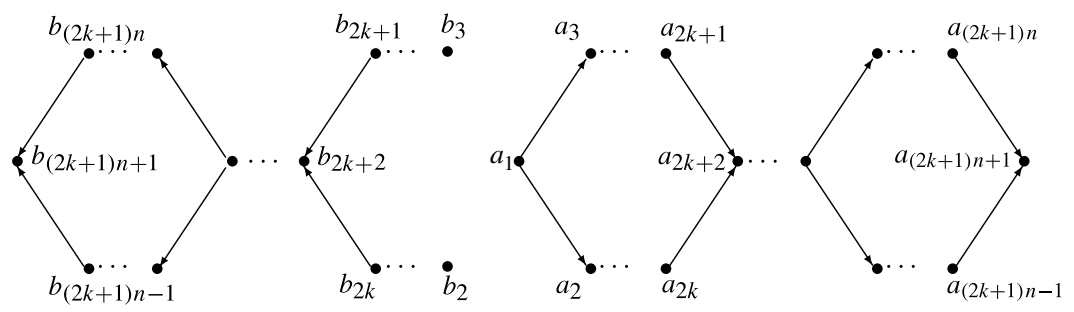

Figure 1. A broken $k$-diamond of length $2 n$.

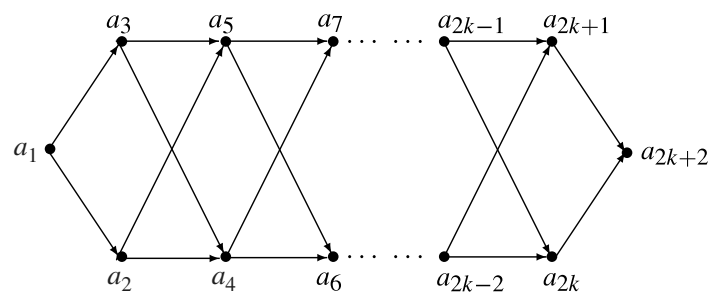

FIGURE 2. A $k$-elongated diamond.

Let $\Delta_{k}(n)$ denote the number of broken $k$-diamond partitions of $n$. From [1], we know that for any positive integer $k$,

$$
\sum_{n=0}^{\infty} \Delta_{k}(n) q^{n}=\frac{f_{2} f_{2 k+1}}{f_{1}^{3} f_{4 k+2}},
$$

where here and throughout this paper, $f_{k}$ is defined by

$$
f_{k}=\prod_{r=1}^{\infty}\left(1-q^{k r}\right) \quad|q|<1 .
$$

Employing generating function manipulations, Andrews and Paule [1] proved that for any integer $n \geq 0$,

$$
\Delta_{1}(2 n+1) \equiv 0(\bmod 3) .
$$

Soon after, Hirschhorn and Sellers [5] found an explicit representation of the generating function for $\Delta_{1}(2 n+1)$ which implied (1.1). Mortenson [7] reproved (1.1) by developing a statistic on the partitions enumerated by $\Delta_{1}(2 n+1)$ which naturally breaks these partitions into three subsets of equal size. Recently, Fu [4] presented a combinatorial proof of (1.1). He also employed his combinatorial approach to naturally define a generalisation of broken $k$-diamond partitions which he termed $k$ dots bracelet partitions. To define $k$ dots bracelet partitions, Fu first gave the definition of the 'infinite bracelet with $k$ dots'. For $k \geq 3$, an 'infinite bracelet with $k$ dots' is the 


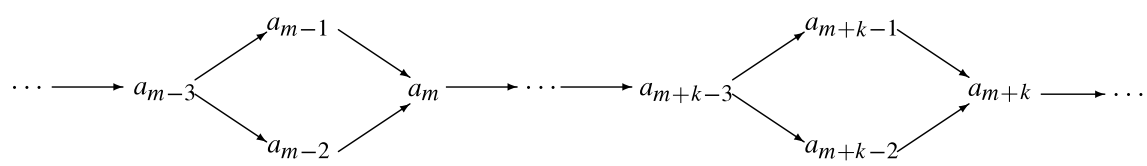

FIGURE 3. Infinite bracelet with $k$ dots.
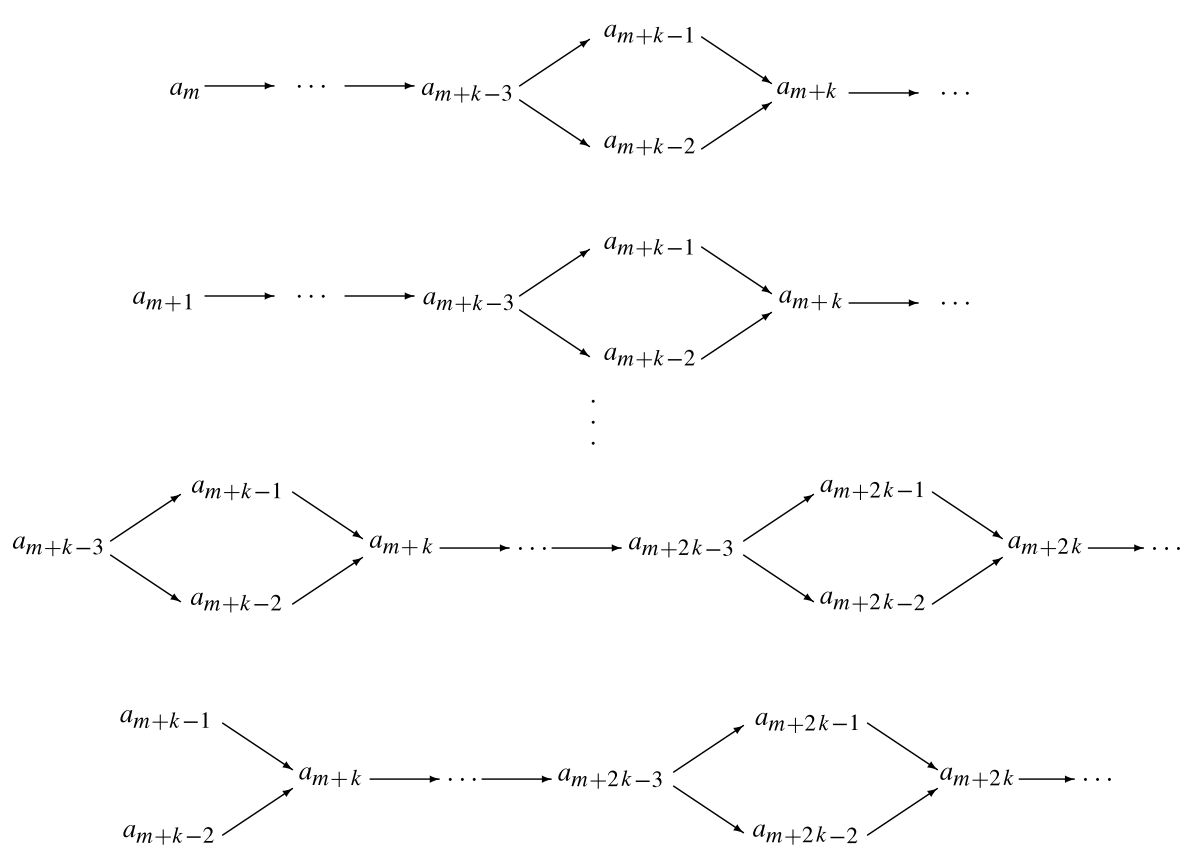

FIGURE 4. $k-1$ different half bracelets.

configuration shown in Figure 3. It is composed of repeating 'diamonds' and 'dots' with $k-2$ 'dots' between two consecutive 'diamonds'.

It is easy to see that there are essentially $k-1$ different ways to cut an infinite bracelet with $k$ dots in half. For each different 'cut', we get a half bracelet (the right half) in different configuration. Graphically, see Figure 4.

For any positive integer $k$, a ' $k$ dots bracelet partition' is a partition which consists of the $k-1$ different half bracelets (in the sense of different configuration) as shown in Figure 4 above. Fu utilised $\mathfrak{B}_{k}(n)$ to denote the number of $k$ dots bracelet partitions of $n$ and proved that

$$
\sum_{n=0}^{\infty} \mathfrak{B}_{k}(n) q^{n}=\frac{f_{2} f_{k}}{f_{1}^{k} f_{2 k}} .
$$

$\mathrm{Fu}$ also established various congruence properties for $\mathfrak{B}_{k}(n)$ modulo primes and modulo powers of 2 . Very recently, Radu and Sellers [8] extended the set of 
congruences proven by $\mathrm{Fu}$ by proving three congruences modulo squares of primes for $\mathfrak{B}_{5}(n), \mathfrak{B}_{7}(n)$ and $\mathfrak{B}_{11}(n)$.

The aim of this paper is to prove some congruences modulo powers of 2 for $\mathfrak{B}_{5}(n)$. The proofs mainly rely on some 2-dissections of certain infinite products. Recall that the $m$-dissection of the power series $P(q)=\sum_{n=0}^{\infty} a_{n} q^{n}$ is the presentation of $P(q)$ as

$$
P(q)=P_{0}(q)+P_{1}(q)+\cdots+P_{m-1}(q),
$$

where

$$
P_{k}(q)=\sum_{n=0}^{\infty} a_{m n+k} q^{m n+k}
$$

The main results of this paper can be stated as follows.

Theorem 1.1. For any integer $n \geq 0$,

$$
\begin{aligned}
\mathfrak{B}_{5}(4 n+3) & \equiv 0\left(\bmod 2^{2}\right), \\
\mathfrak{B}_{5}(8 n+7) & \equiv 0\left(\bmod 2^{4}\right), \\
\mathfrak{B}_{5}(16 n+7) & \equiv 0\left(\bmod 2^{5}\right), \\
\mathfrak{B}_{5}(32 n+31) & \equiv 0\left(\bmod 2^{5}\right) .
\end{aligned}
$$

THEOREM 1.2. Let $n$ be a nonnegative integer. We have

$$
\mathfrak{B}_{5}(32 n+15) \equiv 2^{4} \mathfrak{B}_{5}(8 n+4)\left(\bmod 2^{5}\right) .
$$

If $24 n+11$ is a prime, then

$\mathfrak{B}_{5}(32 n+15)$

$$
\equiv \begin{cases}2^{4}\left(\bmod 2^{5}\right) & \text { if } 24 n+11 \text { is of the form } 11 x^{2}+108 x y+396 y^{2} \\ 0\left(\bmod 2^{5}\right) & \text { or } 44 x^{2}+108 x y+99 y^{2}, \text { where } x \text { and } y \text { are integers }\end{cases}
$$

Theorem 1.3. For all nonnegative integers $n$ and $i=1,2,3,4$,

$$
\begin{aligned}
\mathfrak{B}_{5}(32 n+19) & \equiv 0\left(\bmod 2^{3}\right), \\
\mathfrak{B}_{5}(64 n+51) & \equiv 0\left(\bmod 2^{3}\right), \\
\mathfrak{B}_{5}(64 n+59) & \equiv 0\left(\bmod 2^{3}\right), \\
\mathfrak{B}_{5}(128 n+91) & \equiv 0\left(\bmod 2^{3}\right), \\
\mathfrak{B}_{5}(640 n+128 i+27) & \equiv 0\left(\bmod 2^{3}\right)
\end{aligned}
$$

and

$$
\mathfrak{B}_{5}(64 n+3) \equiv \mathfrak{B}_{5}(640 n+27) \equiv \begin{cases}4\left(\bmod 2^{3}\right) & \text { if } n=P_{k}, \\ 0\left(\bmod 2^{3}\right) & \text { otherwise },\end{cases}
$$

where $P_{k}$ is either of the kth generalised pentagonal numbers $k(3 k \pm 1) / 2$. 
THEOREM 1.4. Let $n$ and $k$ be nonnegative integers. We have

$$
\mathfrak{B}_{5}\left(2^{2 k+3} n+\frac{2^{2 k+3}+1}{3}\right) \equiv \mathfrak{B}_{5}(8 n+3)\left(\bmod 2^{3}\right) .
$$

Theorem 1.5. For all nonnegative integers $n$ and $i=1,2,3,4$,

$$
\mathfrak{B}_{5}(20 n+4 i+2) \equiv \mathfrak{B}_{5}(40 n+22) \equiv 0(\bmod 2)
$$

and

$$
\mathfrak{B}_{5}(40 n+2) \equiv \begin{cases}1(\bmod 2) & \text { if } n=P_{k}, \\ 0(\bmod 2) & \text { otherwise, }\end{cases}
$$

where $P_{k}$ is either of the $k$ th generalised pentagonal numbers $k(3 k \pm 1) / 2$.

THEOREM 1.6. For all nonnegative integers $n$,

$$
\mathfrak{B}_{5}(4 n+1) \equiv b_{5}(n)(\bmod 2),
$$

where $b_{5}(n)$ is the number of 5-regular partitions of $n$.

By Theorem 1.6 and some results proved in $[3,6]$, we can obtain the following corollaries.

COROLlary 1.7. For all nonnegative integers $n, \mathfrak{B}_{5}(8 n+1)$ is odd if and only if $12 n+1$ is a perfect square and $\mathfrak{B}_{5}(16 n+5)$ is even unless $24 n+7=2 x^{2}+5 y^{2}$ for some integers $x$ and $y$.

Corollary 1.8. For all nonnegative integers $n, \mathfrak{B}_{5}(80 n+21), \mathfrak{B}_{5}(80 n+53)$, and $\mathfrak{B}_{5}(4624 n+261)$ are even numbers.

Corollary 1.9. Suppose that $p$ is any prime greater than 3 such that -10 is a quadratic nonresidue modulo $p, u$ is the reciprocal of 24 modulo $p^{2}$, and $p \nmid r$. Then, for all nonnegative integers $n, \mathfrak{B}_{5}\left(16 p^{2} n+16 u(p r-7)+5\right)$ is even.

\section{Two lemmas}

In this section, to prove our main results, we first present two lemmas.

LEMMA 2.1. The following 2-dissection holds

$$
\frac{1}{f_{1}^{4}}=\frac{f_{4}^{14}}{f_{2}^{14} f_{8}^{4}}+4 q \frac{f_{4}^{2} f_{8}^{4}}{f_{2}^{10}} .
$$

Equation (2.1) follows from Entry 25 (v) and (vi) in [2, page 40]. The authors also presented another proof of (2.1) in [11]. 
LEMMA 2.2. The following 2-dissections hold

$$
\frac{f_{5}}{f_{1}}=\frac{f_{8} f_{20}^{2}}{f_{2}^{2} f_{40}}+q \frac{f_{4}^{3} f_{10} f_{40}}{f_{2}^{3} f_{8} f_{20}}
$$

and

$$
\frac{f_{1}}{f_{5}}=\frac{f_{2} f_{8} f_{20}^{3}}{f_{4} f_{10}^{3} f_{40}}-q \frac{f_{4}^{2} f_{40}}{f_{8} f_{10}^{2}} .
$$

Equation (2.2) was proved by Hirschhorn and Sellers in [6]; see also [10, 11]. It is easy to check that for any odd integer $k \geq 1$,

$$
f_{k}(-q)=\frac{f_{2 k}^{3}}{f_{k} f_{4 k}} \text {. }
$$

Replacing $q$ by $-q$ in (2.2) and employing (2.4),

$$
\frac{f_{1} f_{4} f_{10}^{3}}{f_{2}^{3} f_{5} f_{20}}=\frac{f_{8} f_{20}^{2}}{f_{2}^{2} f_{40}}-q \frac{f_{4}^{3} f_{10} f_{40}}{f_{2}^{3} f_{8} f_{20}}
$$

which yields (2.3).

\section{Proofs of the main results}

In this section, we provide proofs of Theorems 1.1-1.6 and Corollaries 1.7-1.9 by employing Lemmas 2.1 and 2.2.

Setting $k=5$ in (1.2),

$$
\sum_{n=0}^{\infty} \mathfrak{B}_{5}(n) q^{n}=\frac{f_{2} f_{5}}{f_{1}^{5} f_{10}}
$$

From (2.1), (2.2) and (3.1), we see that

$$
\begin{aligned}
\sum_{n=0}^{\infty} \mathfrak{B}_{5}(n) q^{n} & =\frac{f_{2}}{f_{10}} \frac{f_{5}}{f_{1}} \frac{1}{f_{1}^{4}}=\frac{f_{2}}{f_{10}}\left(\frac{f_{4}^{14}}{f_{2}^{14} f_{8}^{4}}+4 q \frac{f_{4}^{2} f_{8}^{4}}{f_{2}^{10}}\right)\left(\frac{f_{8} f_{20}^{2}}{f_{2}^{2} f_{40}}+q \frac{f_{4}^{3} f_{10} f_{40}}{f_{2}^{3} f_{8} f_{20}}\right) \\
& =\frac{f_{4}^{14} f_{20}^{2}}{f_{2}^{15} f_{8}^{3} f_{10} f_{40}}+q \frac{f_{4}^{17} f_{40}}{f_{2}^{16} f_{8}^{5} f_{20}}+4 q \frac{f_{4}^{2} f_{8}^{5} f_{20}^{2}}{f_{2}^{11} f_{10} f_{40}}+4 q^{2} \frac{f_{4}^{5} f_{8}^{3} f_{40}}{f_{2}^{12} f_{20}}
\end{aligned}
$$

which yields

$$
\sum_{n=0}^{\infty} \mathfrak{B}_{5}(2 n) q^{n}=\frac{f_{2}^{14} f_{10}^{2}}{f_{1}^{15} f_{4}^{3} f_{5} f_{20}}+4 q \frac{f_{2}^{5} f_{4}^{3} f_{20}}{f_{1}^{12} f_{10}}
$$

and

$$
\sum_{n=0}^{\infty} \mathfrak{B}_{5}(2 n+1) q^{n}=\frac{f_{2}^{17} f_{20}}{f_{1}^{16} f_{4}^{5} f_{10}}+4 \frac{f_{2}^{2} f_{4}^{5} f_{10}^{2}}{f_{1}^{11} f_{5} f_{20}}
$$


In view of (2.1) and (2.3), we deduce that, modulo 32,

$$
\begin{aligned}
& \frac{f_{2}^{17} f_{20}}{f_{1}^{16} f_{4}^{5} f_{10}}+4 \frac{f_{2}^{2} f_{4}^{5} f_{10}^{2}}{f_{1}^{11} f_{5} f_{20}}=\frac{f_{2}^{17} f_{20}}{f_{4}^{5} f_{10}} \frac{1}{f_{1}^{16}}+4 \frac{f_{2}^{2} f_{4}^{5} f_{10}^{2}}{f_{20}} \frac{1}{f_{1}^{12}} \frac{f_{1}}{f_{5}} \\
&=\frac{f_{2}^{17} f_{20}}{f_{4}^{5} f_{10}}\left(\frac{f_{4}^{14}}{f_{2}^{14} f_{8}^{4}}+4 q \frac{f_{4}^{2} f_{8}^{4}}{f_{2}^{10}}\right)^{4}+4 \frac{f_{2}^{2} f_{4}^{5} f_{10}^{2}}{f_{20}}\left(\frac{f_{4}^{14}}{f_{2}^{14} f_{8}^{4}}+4 q \frac{f_{4}^{2} f_{8}^{4}}{f_{2}^{10}}\right)^{3}\left(\frac{f_{2} f_{8} f_{20}^{3}}{f_{4} f_{10}^{3} f_{40}}-q \frac{f_{4}^{2} f_{40}}{f_{8} f_{10}^{2}}\right) \\
& \equiv \frac{f_{2}^{17} f_{20}}{f_{4}^{5} f_{10}}\left(\left(\frac{f_{4}^{14}}{f_{2}^{14} f_{8}^{4}}\right)^{4}+16 q\left(\frac{f_{4}^{14}}{f_{2}^{14} f_{8}^{4}}\right)^{3} \frac{f_{4}^{2} f_{8}^{4}}{f_{2}^{10}}\right) \\
&+4 \frac{f_{2}^{2} f_{4}^{5} f_{10}^{2}}{f_{20}}\left(\left(\frac{f_{4}^{14}}{f_{2}^{14} f_{8}^{4}}\right)^{3}+12 q\left(\frac{f_{4}^{14}}{f_{2}^{14} f_{8}^{4}}\right)^{2} \frac{f_{4}^{2} f_{8}^{4}}{f_{2}^{10}}\right)\left(\frac{f_{2} f_{8} f_{20}^{3}}{f_{4} f_{10}^{3} f_{40}}-q \frac{f_{4}^{2} f_{40}}{f_{8} f_{10}^{2}}\right) \\
& \equiv \frac{f_{4}^{51} f_{20}}{f_{2}^{39} f_{8}^{16} f_{10}}+4 \frac{f_{4}^{46} f_{20}^{2}}{f_{2}^{39} f_{8}^{11} f_{10} f_{40}}+16 q \frac{f_{4}^{39} f_{20}}{f_{2}^{35} f_{8}^{8} f_{10}} \\
&-4 q \frac{f_{4}^{49} f_{40}}{f_{2}^{40} f_{8}^{13} f_{20}}+16 q \frac{f_{4}^{34} f_{20}^{2}}{f_{2}^{35} f_{8}^{3} f_{10} f_{40}}-16 q^{2} \frac{f_{4}^{37} f_{40}}{f_{2}^{36} f_{8}^{5} f_{20}} .
\end{aligned}
$$

Combining (3.3) and (3.4), we find that, modulo 32,

$$
\sum_{n=0}^{\infty} \mathfrak{B}_{5}(4 n+3) q^{n} \equiv 16 \frac{f_{2}^{39} f_{10}}{f_{1}^{35} f_{4}^{8} f_{5}}-4 \frac{f_{2}^{49} f_{20}}{f_{1}^{40} f_{4}^{13} f_{10}}+16 \frac{f_{2}^{34} f_{10}^{2}}{f_{1}^{35} f_{4}^{3} f_{5} f_{20}}
$$

Congruence (1.3) follows from (3.5).

Employing (2.1) and (2.3), we have, modulo 8,

$$
\begin{aligned}
& 4 \frac{f_{2}^{39} f_{10}}{f_{4}^{8}} \frac{1}{f_{1}^{36}} \frac{f_{1}}{f_{5}}-\frac{f_{2}^{49} f_{20}}{f_{4}^{13} f_{10}} \frac{1}{f_{1}^{40}}+4 \frac{f_{2}^{34} f_{10}^{2}}{f_{4}^{3} f_{20}} \frac{1}{f_{1}^{36}} \frac{f_{1}}{f_{5}} \\
& =4 \frac{f_{2}^{39} f_{10}}{f_{4}^{8}}\left(\frac{f_{4}^{14}}{f_{2}^{14} f_{8}^{4}}+4 q \frac{f_{4}^{2} f_{8}^{4}}{f_{2}^{10}}\right)^{9}\left(\frac{f_{2} f_{8} f_{20}^{3}}{f_{4} f_{10}^{3} f_{40}}-q \frac{f_{4}^{2} f_{40}}{f_{8} f_{10}^{2}}\right)-\frac{f_{2}^{49} f_{20}}{f_{4}^{13} f_{10}}\left(\frac{f_{4}^{14}}{f_{2}^{14} f_{8}^{4}}+4 q \frac{f_{4}^{2} f_{8}^{4}}{f_{2}^{10}}\right)^{10} \\
& +4 \frac{f_{2}^{34} f_{10}^{2}}{f_{4}^{3} f_{20}}\left(\frac{f_{4}^{14}}{f_{2}^{14} f_{8}^{4}}+4 q \frac{f_{4}^{2} f_{8}^{4}}{f_{2}^{10}}\right)^{9}\left(\frac{f_{2} f_{8} f_{20}^{3}}{f_{4} f_{10}^{3} f_{40}}-q \frac{f_{4}^{2} f_{40}}{f_{8} f_{10}^{2}}\right) \\
& \equiv 4 \frac{f_{2}^{39} f_{10}}{f_{4}^{8}}\left(\frac{f_{4}^{14}}{f_{2}^{14} f_{8}^{4}}\right)^{9}\left(\frac{f_{2} f_{8} f_{20}^{3}}{f_{4} f_{10}^{3} f_{40}}-q \frac{f_{4}^{2} f_{40}}{f_{8} f_{10}^{2}}\right)-\frac{f_{2}^{49} f_{20}}{f_{4}^{13} f_{10}}\left(\frac{f_{4}^{14}}{f_{2}^{14} f_{8}^{4}}\right)^{10} \\
& +4 \frac{f_{2}^{34} f_{10}^{2}}{f_{4}^{3} f_{20}}\left(\frac{f_{4}^{14}}{f_{2}^{14} f_{8}^{4}}\right)^{9}\left(\frac{f_{2} f_{8} f_{20}^{3}}{f_{4} f_{10}^{3} f_{40}}-q \frac{f_{4}^{2} f_{40}}{f_{8} f_{10}^{2}}\right) \\
& \equiv 4 \frac{f_{4}^{117} f_{20}^{3}}{f_{2}^{86} f_{8}^{35} f_{10}^{2} f_{40}}-4 q \frac{f_{4}^{120} f_{40}}{f_{2}^{87} f_{8}^{37} f_{10}}-\frac{f_{4}^{127} f_{20}}{f_{2}^{91} f_{8}^{40} f_{10}}+4 \frac{f_{4}^{122} f_{20}^{2}}{f_{2}^{91} f_{8}^{35} f_{10} f_{40}}-4 q \frac{f_{4}^{125} f_{40}}{f_{2}^{92} f_{8}^{37} f_{20}} \text {. }
\end{aligned}
$$


Combining (3.5) and (3.6), we see that, modulo 32,

$$
\sum_{n=0}^{\infty} \mathfrak{B}_{5}(8 n+7) q^{n} \equiv 16 \frac{f_{2}^{120} f_{20}}{f_{1}^{87} f_{4}^{37} f_{5}}+16 \frac{f_{2}^{125} f_{20}}{f_{1}^{92} f_{4}^{37} f_{10}} .
$$

Congruence (1.4) follows from (3.7).

By (2.1) and (2.3), we have, modulo 2,

$$
\begin{aligned}
\frac{f_{2}^{120} f_{20}}{f_{4}^{37}} & \frac{1}{f_{1}^{88}} \frac{f_{1}}{f_{5}}+\frac{f_{2}^{125} f_{20}}{f_{1}^{92} f_{4}^{37} f_{10}} \\
= & \frac{f_{2}^{120} f_{20}}{f_{4}^{37}}\left(\frac{f_{4}^{14}}{f_{2}^{14} f_{8}^{4}}+4 q \frac{f_{4}^{2} f_{8}^{4}}{f_{2}^{10}}\right)^{22}\left(\frac{f_{2} f_{8} f_{20}^{3}}{f_{4} f_{10}^{3} f_{40}}-q \frac{f_{4}^{2} f_{40}}{f_{8} f_{10}^{2}}\right) \\
& +\frac{f_{2}^{125} f_{20}}{f_{4}^{37} f_{10}}\left(\frac{f_{4}^{14}}{f_{2}^{14} f_{8}^{4}}+4 q \frac{f_{4}^{2} f_{8}^{4}}{f_{2}^{10}}\right)^{23} \\
\equiv & \frac{f_{2}^{120} f_{20}}{f_{4}^{37}}\left(\frac{f_{4}^{14}}{f_{2}^{14} f_{8}^{4}}\right)^{22}\left(\frac{f_{2} f_{8} f_{20}^{3}}{f_{4} f_{10}^{3} f_{40}}-q \frac{f_{4}^{2} f_{40}}{f_{8} f_{10}^{2}}\right)+\frac{f_{2}^{125} f_{20}}{f_{4}^{37} f_{10}}\left(\frac{f_{4}^{14}}{f_{2}^{14} f_{8}^{4}}\right)^{23} \\
\equiv & \frac{f_{4}^{270} f_{20}^{4}}{f_{2}^{187} f_{8}^{87} f_{10}^{3} f_{40}}-q \frac{f_{4}^{273} f_{20} f_{40}}{f_{2}^{188} f_{8}^{89} f_{10}^{2}}+\frac{f_{4}^{285} f_{20}}{f_{2}^{197} f_{8}^{92} f_{10}} .
\end{aligned}
$$

It follows from (3.7) and (3.8) that, modulo 32,

$$
\sum_{n=0}^{\infty} \mathfrak{B}_{5}(16 n+7) q^{n} \equiv 16 \frac{f_{2}^{270} f_{10}^{4}}{f_{1}^{187} f_{4}^{87} f_{5}^{3} f_{20}}+16 \frac{f_{2}^{285} f_{10}}{f_{1}^{197} f_{4}^{92} f_{5}}
$$

and

$$
\sum_{n=0}^{\infty} \mathfrak{B}_{5}(16 n+15) q^{n} \equiv 16 \frac{f_{2}^{273} f_{10} f_{20}}{f_{1}^{188} f_{4}^{89} f_{5}^{2}} .
$$

It is easy to see that for any positive integer $k$, modulo 2 ,

$$
f_{k}^{2} \equiv f_{2 k}
$$

Employing (3.11), we deduce that, modulo 2,

$$
\begin{gathered}
\frac{f_{2}^{270} f_{10}^{4}}{f_{1}^{187} f_{4}^{87} f_{5}^{3} f_{20}} \equiv f_{1}^{5} f_{5}, \\
\frac{f_{2}^{285} f_{10}}{f_{1}^{197} f_{4}^{92} f_{5}} \equiv f_{1}^{5} f_{5}
\end{gathered}
$$

and

$$
\frac{f_{2}^{273} f_{10} f_{20}}{f_{1}^{188} f_{4}^{89} f_{5}^{2}} \equiv f_{2} f_{20}
$$


From (3.9), (3.12) and (3.13), we see that, modulo 32,

$$
\sum_{n=0}^{\infty} \mathfrak{B}_{5}(16 n+7) q^{n} \equiv 0
$$

Congruence (1.5) follows from (3.15).

In view of (3.10) and (3.14), we obtain, modulo 32,

$$
\sum_{n=0}^{\infty} \mathfrak{B}_{5}(32 n+15) q^{n} \equiv 16 f_{1} f_{10}
$$

and

$$
\sum_{n=0}^{\infty} \mathfrak{B}_{5}(32 n+31) q^{n} \equiv 0
$$

which yields (1.6).

Let $c(n)$ be defined by

$$
f_{1} f_{10}=1+\sum_{n=1}^{\infty} c(n) q^{n}
$$

In [9], Sun proved that if $24 n+11$ is a prime, then

$$
c(n)= \begin{cases}1 & \text { if } 24 n+11 \text { is of the form } 11 x^{2}+108 x y+396 y^{2} \\ -1 & \text { if } 24 n+11 \text { is of the form } 44 x^{2}+108 x y+99 y^{2} \\ 0 & \text { otherwise }\end{cases}
$$

Congruence (1.8) follows from (3.16), (3.17) and (3.18).

By (3.5) and (3.11), we find that, modulo 8,

$$
\sum_{n=0}^{\infty} \mathfrak{B}_{5}(4 n+3) q^{n} \equiv 4 \frac{f_{2}^{49} f_{20}}{f_{1}^{40} f_{4}^{13} f_{10}} \equiv 4 \frac{f_{8} f_{10}}{f_{2}},
$$

which yields

$$
\sum_{n=0}^{\infty} \mathfrak{B}_{5}(8 n+3) q^{n} \equiv 4 \frac{f_{4} f_{5}}{f_{1}}
$$

It follows from (2.2) and (3.11) that, modulo 2,

$$
\begin{aligned}
\frac{f_{4} f_{5}}{f_{1}} & =f_{4}\left(\frac{f_{8} f_{20}^{2}}{f_{2}^{2} f_{40}}+q \frac{f_{4}^{3} f_{10} f_{40}}{f_{2}^{3} f_{8} f_{20}}\right)=\frac{f_{4} f_{8} f_{20}^{2}}{f_{2}^{2} f_{40}}+q \frac{f_{4}^{4} f_{10} f_{40}}{f_{2}^{3} f_{8} f_{20}} \\
& \equiv f_{8}+q \frac{f_{2} f_{40}}{f_{10}} .
\end{aligned}
$$

By (3.19) and (3.20), we deduce that, modulo 8,

$$
\sum_{n=0}^{\infty} \mathfrak{B}_{5}(16 n+3) q^{n} \equiv 4 f_{4}
$$


and

$$
\sum_{n=0}^{\infty} \mathfrak{B}_{5}(16 n+11) q^{n} \equiv 4 \frac{f_{1} f_{20}}{f_{5}}
$$

Congruences (1.9) and (1.10) follow from (3.21).

In view of (2.3) and (3.11), we have, modulo 2,

$$
\begin{aligned}
\frac{f_{1} f_{20}}{f_{5}} & =f_{20}\left(\frac{f_{2} f_{8} f_{20}^{3}}{f_{4} f_{10}^{3} f_{40}}-q \frac{f_{4}^{2} f_{40}}{f_{8} f_{10}^{2}}\right)=\frac{f_{2} f_{8} f_{20}^{4}}{f_{4} f_{10}^{3} f_{40}}-q \frac{f_{4}^{2} f_{20} f_{40}}{f_{8} f_{10}^{2}} \\
& \equiv \frac{f_{8} f_{10}}{f_{2}}-q f_{40} .
\end{aligned}
$$

Employing (3.22) and (3.23), we find that, modulo 8,

$$
\sum_{n=0}^{\infty} \mathfrak{B}_{5}(32 n+11) q^{n} \equiv 4 \frac{f_{4} f_{5}}{f_{1}}
$$

and

$$
\sum_{n=0}^{\infty} \mathfrak{B}_{5}(32 n+27) q^{n} \equiv 4 f_{20}
$$

Congruences (1.11), (1.12) and (1.13) follow from (3.25).

In view of (3.21) and (3.25), we have, modulo 8,

$$
\sum_{n=0}^{\infty} \mathfrak{B}_{5}(64 n+3) q^{n} \equiv \sum_{n=0}^{\infty} \mathfrak{B}_{5}(640 n+27) q^{n} \equiv 4 f_{1} .
$$

By Euler's pentagonal number theorem,

$$
f_{1}=\sum_{k=-\infty}^{\infty}(-1)^{k} q^{k(3 k-1) / 2}=1+\sum_{k=1}^{\infty}(-1)^{k}\left(q^{k(3 k-1) / 2}+q^{k(3 k+1) / 2}\right) .
$$

Combining (3.26) and (3.27), we deduce that, modulo 8,

$$
\begin{aligned}
\sum_{n=0}^{\infty} \mathfrak{B}_{5}(64 n+3) q^{n} & \equiv \sum_{n=0}^{\infty} \mathfrak{B}_{5}(640 n+27) q^{n} \equiv 4+4 \sum_{k=1}^{\infty}(-1)^{k}\left(q^{k(3 k-1) / 2}+q^{k(3 k+1) / 2}\right) \\
& \equiv 4+4 \sum_{k=1}^{\infty}\left(q^{k(3 k-1) / 2}+q^{k(3 k+1) / 2}\right),
\end{aligned}
$$

which yields (1.14).

From (3.19) and (3.24), we see that, modulo 8,

$$
\mathfrak{B}_{5}(32 n+11) \equiv \mathfrak{B}_{5}(8 n+3) .
$$

By (3.28) and mathematical induction, we find that (1.15) is true. 
By (2.3), (3.2) and (3.11), we see that, modulo 2,

$$
\sum_{n=0}^{\infty} \mathfrak{B}_{5}(2 n) q^{n} \equiv \frac{f_{1}}{f_{5}} \equiv \frac{f_{2} f_{8} f_{20}^{3}}{f_{4} f_{10}^{3} f_{40}}+q \frac{f_{4}^{2} f_{40}}{f_{8} f_{10}^{2}} \equiv \frac{f_{2} f_{4}}{f_{10}}+q f_{20},
$$

which implies that, modulo 2 ,

$$
\sum_{n=0}^{\infty} \mathfrak{B}_{5}(4 n) q^{n} \equiv \frac{f_{1} f_{2}}{f_{5}}
$$

and

$$
\sum_{n=0}^{\infty} \mathfrak{B}_{5}(4 n+2) q^{n} \equiv f_{10}
$$

Theorem 1.5 follows from (3.27) and (3.29).

Employing (2.3) and (3.11), we obtain that, modulo 2,

$$
\begin{aligned}
\frac{f_{1} f_{2}}{f_{5}} & =f_{2}\left(\frac{f_{2} f_{8} f_{20}^{3}}{f_{4} f_{10}^{3} f_{40}}-q \frac{f_{4}^{2} f_{40}}{f_{8} f_{10}^{2}}\right) \\
& =\frac{f_{2}^{2} f_{8} f_{20}^{3}}{f_{4} f_{10}^{3} f_{40}}-q \frac{f_{2} f_{4}^{2} f_{40}}{f_{8} f_{10}^{2}} \equiv \frac{f_{8}}{f_{10}}+q f_{2} f_{20},
\end{aligned}
$$

which implies that, modulo 2 ,

$$
\sum_{n=0}^{\infty} \mathfrak{B}_{5}(8 n+4) q^{n} \equiv f_{1} f_{10}
$$

Congruence (1.7) follows from (3.16) and (3.30).

By (3.3), (3.4) and (3.11), we find that, modulo 2,

$$
\sum_{n=0}^{\infty} \mathfrak{B}_{5}(4 n+1) q^{n} \equiv \frac{f_{2}^{51} f_{10}}{f_{1}^{39} f_{4}^{16} f_{5}} \equiv \frac{f_{5}}{f_{1}} .
$$

Let $b_{5}(n)$ denote the number of 5-regular partitions of $n$. Adopting the convention that $b_{5}(0)=1$, the generating function for $b_{5}(n)$ is then

$$
\sum_{n=0}^{\infty} b_{5}(n) q^{n}=\frac{f_{5}}{f_{1}}
$$

Theorem 1.6 follows from (3.31) and (3.32).

Using the theory of modular forms, Calkin et al. [3] proved a result equivalent to the following: for all integers $n \geq 0, b_{5}(2 n)$ is odd if and only if $12 n+1$ is a perfect square. Employing nothing more than Jacobi's triple product identity, Hirschhorn and Sellers [6] proved the following result: for all integers $n \geq 0, b_{5}(4 n+1)$ is even unless $24 n+7=2 x^{2}+5 y^{2}$ for some integers $x$ and $y$.

Corollary 1.7 follows from these two results and Theorem 1.6. 
In [3], Calkin et al. also proved that for all integers $n \geq 0$, modulo 2 ,

$$
b_{5}(20 n+5) \equiv 0
$$

and

$$
b_{5}(20 n+13) \equiv 0 \text {. }
$$

Hirschhorn and Sellers [6] discovered that, modulo 2,

$$
b_{5}(1156 n+65) \equiv 0 .
$$

Corollary 1.8 follows from (3.33), (3.34), (3.35) and Theorem 1.6.

Hirschhorn and Sellers [6] also proved the following result: Suppose that $p$ is any prime greater than 3 such that -10 is a quadratic nonresidue modulo $p, u$ is the reciprocal of 24 modulo $p^{2}$, and $p \nmid r$. Then, for all integers $n$, modulo 2 ,

$$
b_{5}\left(4 p^{2} n+4 u(p r-7)+1\right) \equiv 0 .
$$

Corollary 1.9 follows from the above result and Theorem 1.6. This completes the proof.

\section{Acknowledgements}

The authors are grateful to the editor and the anonymous referee for their valuable suggestions, corrections and comments which resulted in a great improvement of the original manuscript.

\section{References}

[1] G. E. Andrews and P. Paule, 'MacMahon's partition analysis XI: broken diamonds and modular forms', Acta Arith. 126 (2007), 281-294.

[2] B. C. Berndt, Ramanujan's Notebooks, Part III (Springer, New York, 1991).

[3] N. Calkin, N. Drake, K. James, S. Law, P. Lee, D. Penniston and J. Radder, 'Divisibility properties of the 5-regular and 13-regular partition functions', Integers 8 (2008), \#A60.

[4] S. Fu, 'Combinatorial proof of one congruence for the broken 1-diamond partition and a generalization', Int. J. Number Theory 7 (2011), 133-144.

[5] M. D. Hirschhorn and J. A. Sellers, 'On recent congruence results of Andrews and Paule', Bull. Aust. Math. Soc. 75 (2007), 121-126.

[6] M. D. Hirschhorn and J. A. Sellers, 'Elementary proofs of parity results for 5-regular partitions', Bull. Aust. Math. Soc. 81 (2010), 58-63.

[7] E. Mortenson, 'On the broken 1-diamond partition', Int. J. Number Theory 4 (2008), 199-218.

[8] S. Radu and J. A. Sellers, 'Congruences modulo squares of primes for Fu's $k$ dots bracelet partitions', Int. J. Number Theory 9 (2013), 939-943.

[9] Z. H. Sun, 'The expansion of $\prod_{k=1}^{\infty}\left(1-q^{a k}\right)\left(1-q^{b k}\right)$ ', Acta Arith. 134 (2008), 11-29.

[10] E. X. W. Xia and O. X. M. Yao, 'Some modular relations for the Göllnitz-Gordon functions by an even-odd method', J. Math. Anal. Appl. 387 (2012), 126-138.

[11] E. X. W. Xia and O. X. M. Yao, 'Analogues of Ramanujan's partition identities', Ramanujan J. 31 (2013), 373-396. 
ERNEST X. W. XIA, Department of Mathematics, Jiangsu University, Jiangsu, Zhenjiang 212013, PR China

e-mail: Ernestxwxia@163.com

OLIVIA X. M. YAO, Department of Mathematics, Jiangsu University, Jiangsu, Zhenjiang 212013, PR China

e-mail: yaoxiangmei@163.com 